\title{
Vision-based Localization of Leader-Follower Formations
}

\author{
Gian Luca Mariottini, George Pappas, Domenico Prattichizzo, Kostas Daniilidis
}

\begin{abstract}
This paper focuses on the localization problem for a mobile camera network. In particular, we consider the case of leader-follower formations of nonholonomic mobile vehicles equipped with vision sensors which provide only the bearing to the other robots. We prove a sufficient condition for observability and show that recursive estimation enables a leader-follower formation if the leader is not trapped in an unobservable configuration. We employ an Extended Kalman Filter for the estimation of each follower position and orientation with respect to the leader and we adopt a feedback linearizing control strategy to achieve a desired formation. Simulation results in a noisy environment are provided.
\end{abstract}

\section{INTRODUCTION}

Distributed vision systems or camera networks are widely employed today in order to monitor the environment. Deployment of camera networks can be static at fixed positions or on mobile platforms in formations, possibly guided by a leader. On the other hand, collaborative sensing might not be the ultimate goal and just needed for the navigation of a formation of robots. In all cases where a cooperation between robots exists, we need to solve the localization problem: find the relative position and orientation of all robots with respect to a reference coordinate system. In the case of formation navigation, this is needed because the desired positions might be with respect to a common frame. In the case of camera-sensor networks, we need to relate spatial information gathered by one sensor to the rest of the network in order to support location-aware applications and facilitate exploration and map building. The common ingredient in all these problems is the use of passive vision systems -offthe-shelf cameras- which can provide only the projection of points in the world. This is dual to wireless networks where only distance or some function of distance (inverse square) can be measured.

In this paper, we address the case of leader-follower formations of nonholonomic ground vehicles each one equipped with a camera which provides only the bearing to the other robots. We explicitly list here the assumptions we make about sensing, communication, and control:

- Sensing: We assume each robot equipped with a calibrated vision sensor (e.g. a panoramic camera). The bearing is obtained through the reciprocal observation of cameras. A colored marker placed on the follower

Research at the University of Pennsylvania has been partially supported by the ARO MURI DAAD 19-02-01-0383 and NSF ITR 0324977.

G.L. Mariottini and D. Prattichizzo are with Dipartimento di Ingegneria dell'Informazione, University of Siena, Via Roma 56, 53100 Siena, Italy \{gmariottini, prattichizzo\}edii.unisi.it

G. Pappas and K. Daniilidis are with the GRASP Laboratory, University of Pennsylvania, Philadelphia, PA 19104, USA $\{$ kostas, pappasg\}@grasp. upenn . edu is observed by the cameras and will help to detect the line of sight. The robots cannot see other points on the environment.

- Communication: All computation is carried out on the leader, who receives the measurements from all followers, and transmits the control velocities to all followers.

- Actuation and control: Vehicle kinematics obey the unicycle model. The controller's goal is to keep a desired position and orientation with respect to the leader.

The problem is to find the relative position and orientation with respect to the leader so that it can be provided as feedback to the control loop or so that all information gathered by the entire network can be expressed with respect to a single reference coordinate frame, the one of the leader. We conduct a change of variables so that the resulting measurement equation is linear but the plant is nonlinear. We prove a sufficient condition for observability depending only on the bearing rate of change (optical flow) and the leader's angular velocity. Then we employ an Extended Kalman Filter for the estimation of position and orientation of each follower with respect to the leader. We used the estimated state as feedback in achieving a desired relative pose for each follower using feedback linearization. The contribution of this paper is twofold: analytical because we prove a condition sufficient for observability, and constructive because we show that recursive estimation enables a leader-follower formation if the leader is not stuck in an unobservable set-up. It is the first time in the literature that the localization problem for a mobile camera network is solved using only bearing.

The paper is organized as follows. In Sec. II we recall notions of nonlinear observability. In Sec. III the general leader-follower kinematic model is presented together with a discussion on the communication network. In Sec. IV we present an analytical formulation of observability for the leader-formation robot setup. Sec. V reports the description of the nonlinear observer and of the leader-follower control law. Simulation results are presented in Sec. VI for noisy measurements. Finally, in Sec. VII the major contributions of the paper are summarized.

\section{A. Previous work}

The problem of mobile robot localization using optical information is intrinsically nonlinear [2], in fact linearized approximations can be non-observable, while tools from differential nonlinear system theory prove the possibility to reconstruct the robust state. This localization problem is often referred to as the observability of perspective dynamical systems [10] [9] and can be embedded in the more 
general problem of current state estimation using inputoutput measurement. In [4] the current state estimation for single robot is approached using a local Luembergerlike nonlinear observer, based on projections of stationary landmarks in the environment. Other mobile approaches use range measurements [3], [5]. Grabowski and Khosla [7] as well as Doherty et al. [6] use range only measurements in mobile setups. In [1], a function of the Fisher Information Matrix resulting from the probability density function of multiple range measurements is maximized with respect to motions of the network nodes so that uncertainty in locations is minimized. In stationary setups, localization from bearing only has been studied in [15], [17]. Moore et al. [14] use only range measurements and solve the stationary case as a graph realization problem.

\section{OBSERVABILITY FOR NONLINEAR SYSTEMS}

In order to approach the observability study for multi nonholonomic robots in a leader-follower configuration equipped with omnidirectional cameras, we present some basic facts about observability for nonlinear systems [11] [12]. We then derive Proposition 2 that will be used in characterizing the observability in our multi-robot context.

Consider a vector $\mathbf{s} \triangleq\left[s_{1}, s_{2}, \ldots, s_{n}\right]^{T} \in \mathbb{R}^{n}$. For a scalarvalued function $\lambda: \mathbb{R}^{n} \mapsto \mathbb{R}$ and a vector-valued $f: \mathbb{R}^{n} \mapsto$ $\mathbb{R}^{n}$ we define the two following operators:

$$
\begin{gathered}
d \lambda(\mathbf{s}) \triangleq \frac{\partial \lambda(\mathbf{s})}{\partial \mathbf{s}}=\left[\frac{\partial \lambda(\mathbf{s})}{\partial s_{1}}, \frac{\partial \lambda(\mathbf{s})}{\partial s_{2}}, \ldots \frac{\partial \lambda(\mathbf{s})}{\partial s_{n}}\right] \\
L_{f} \lambda(\mathbf{s}) \triangleq \frac{\partial \lambda(\mathbf{s})}{\partial \mathbf{s}} f(\mathbf{s})=\sum_{k=1}^{n} \frac{\partial \lambda(\mathbf{s})}{\partial s_{k}} f_{k}(\mathbf{s})
\end{gathered}
$$

where the $L_{f} \lambda(\mathbf{s})$ is called Lie derivative of $\lambda$ along $f$.

Higher Lie derivatives $L_{f}^{i} \lambda(\mathbf{s})(i=0,1,2, \ldots, n)$ are recursively defined as [10]:

$$
L_{f}^{0} \lambda(\mathbf{s}) \triangleq \lambda(\mathbf{s}), \quad L_{f}^{i} \lambda(\mathbf{s}) \triangleq\left(\frac{\partial}{\partial \mathbf{s}} L_{f}^{i-1} \lambda(\mathbf{s})\right) f(\mathbf{s}) .
$$

Consider now a generic nonlinear system $\Sigma_{N}$ of the form

$$
\Sigma_{N}:\left\{\begin{array}{l}
\dot{\mathbf{s}}(t)=\mathbf{f}(\mathbf{s}(t), \mathbf{u}(t)), \mathbf{s}(0)=\mathbf{s}_{0} \in \mathbb{R}^{n} \\
\mathbf{y}(t) \triangleq \mathbf{h}(\mathbf{s}(t))=\left[h_{1}, h_{2}, \ldots h_{m}\right]^{T}
\end{array}\right.
$$

where $\mathbf{s}(t) \triangleq\left[s_{1}(t), s_{2}(t), \ldots, s_{n}(t)\right]^{T} \in \mathcal{S}$ is the system state, $\mathbf{y}(t) \in \mathcal{Y}$ is the observation vector and $\mathbf{u}(t) \in \mathcal{U}$ is the input to the system. $\mathcal{S}, \mathcal{Y}$ and $\mathcal{U}$ are differential manifolds of dimension $n, m$ and $p$, respectively.

The problem of observability for this kind of systems can be roughly viewed as the injectivity, with respect to the initial condition, of the input-output map $\mathcal{R}_{\Sigma_{N}}: \mathcal{S} \times \mathcal{U} \mapsto \mathcal{Y}$. Two states $\mathbf{s}_{1}$ and $\mathbf{s}_{2}$ are indistinguishable [16] $\left(\mathbf{s}_{1} I \mathbf{s}_{2}\right)$, if $\mathbf{y}_{s_{1}, u(t)}=\mathbf{y}_{s_{2}, u(t)}$, i.e. there exists an input $\mathbf{u}(t)$ and a time $t$ for which, starting from different $\mathbf{s}_{1}$ and $\mathbf{s}_{2}$ then the system (2) exhibits the same outputs.

Observability and observation space concepts directly follow from that one of indistinguishability.

Definition 1 (Observability [16]): Given two states $\mathbf{s}_{1}, \mathbf{s}_{2} \in \mathcal{S}$, a system $\Sigma_{N}$ as in (2) is observable if we have

$$
\mathbf{s}_{1} I \mathbf{s}_{2} \Rightarrow \mathbf{s}_{1}=\mathbf{s}_{2}
$$

Definition 2 (Observation Space [16]): The observation space of $\Sigma_{N}$ is defined as:

$$
H=\operatorname{span}_{\mathbb{R}}\left\{L_{f}^{j-1} h_{k}(\mathbf{s}) \mid k=1, \ldots, m ; j=1, \ldots, j_{m}\right\}
$$

where $j_{1}, j_{2}, \ldots j_{m}$ are positive integers satisfying $j_{1}+j_{2}+$ $\ldots+j_{m}=n$.

From these two definitions the following proposition follows:

Proposition 1 (Observability rank condition [13]): The system in (2) is said to be observable at a point $\hat{\mathbf{s}} \in \mathbb{R}^{n}$, if there exist an open set $U \subset \mathbb{R}^{n}$ of $\hat{\mathbf{s}}$ and positive integers $j_{1}, j_{2}, \ldots, j_{m}$ satisfying $j_{1}+j_{2}+\ldots+j_{m}=n$ such that, for arbitrary $\mathbf{s} \in U$ the set of row vectors defined by

$$
\mathcal{H}=\left\{L_{f}^{j-1} d h_{i}(\mathbf{s}) \mid i=1, \ldots, m ; j=1, \ldots, j_{m}\right\}
$$

is linearly independent.

It is important to note that the above proposition is a sufficient condition i.e. we can still have that the codistribution $\mathcal{H}$ is rank deficient but the system is still observable. Note moreover that, for general nonlinear systems, global or complete observability can not be usually expected. Due to this fact local observability would be suitable notions. Note however that the above proposition implies the local weak observability [8].

Condition (3) can be also tested by checking the full rank of the so-called Extended Output Jacobian matrix J [4]. In the following Proposition we give a formal proof of this assertion.

Proposition 2: Consider the generic nonlinear system $\Sigma_{N}$ as in (2). Then the observability condition 3 in Prop. 1 is equivalent to the set of row vectors $(j$ is the order of differentiation):

$$
\left\{d h_{i}^{(j-1)}(\mathbf{s}) \mid i=1, \ldots, m ; j=1, \ldots, n\right\}
$$

being linearly independent. From (4) we define the Extended Output Jacobian (EOJ) matrix $\mathbf{J} \in \mathbb{R}^{m n \times n}$, built by stacking the rows in (4).

Proof: The proof is constructive. Computing the Lie derivatives in (3) and from (1) it results that $(\forall i=1, \ldots, m)$,

$$
\begin{aligned}
& k=1 \quad: \quad L_{f}^{0} d h_{i}(\mathbf{s})=\frac{\partial h_{i}}{\partial \mathbf{x}}(\mathbf{s})=d h_{i}^{(0)}(\mathbf{s}) \\
& k=2 \quad: \quad L_{f}^{1} d h_{i}(\mathbf{s})=\frac{\partial}{\partial \mathbf{s}}\left(L_{f}^{0} d h_{i}(\mathbf{s})\right) f(\mathbf{s})= \\
& =d\left(\frac{\partial h_{i}}{\partial \mathbf{s}} f(\mathbf{s})\right)=d\left(\frac{\partial h_{i}}{\partial \mathbf{s}} \frac{\partial \mathbf{s}}{\partial t}\right)=d h_{i}^{(1)}(\mathbf{s}) \\
& k=3 \quad: \quad L_{f}^{2} d h_{i}(\mathbf{s})=d\left(\frac{\partial}{\partial \mathbf{s}}\left(L_{f}^{1} h_{i}\right) f(\mathbf{s})\right)= \\
& \left.=d\left(\frac{\partial h_{i}^{(1)}}{\partial \mathbf{s}} \dot{\mathbf{s}}\right)=d\left[\frac{\partial\left(\frac{\partial h_{i}}{\partial t}\right)}{\partial \mathbf{s}} \frac{\partial \mathbf{s}}{\partial t}\right]=d h_{i}^{(2)}(\mathbf{s}\rangle 7\right) \\
& \begin{array}{rcc}
\vdots & \vdots & \vdots \\
k=n & \vdots & L_{f}^{n-1} d h_{i}(\mathbf{s})=d h_{i}^{(n-1)}(\mathbf{s})
\end{array}
\end{aligned}
$$

and by stacking (5),(6),(7) and (8) in a matrix, from Prop. 1 we obtain the thesis. 
Remark 1: Roughly speaking the above proposition suggests testing the observability of a nonlinear system simply checking the rank of a matrix made of the state partial derivative of the output vector and of all its $n-1$ time derivatives. Moreover, from Prop. 2, it is straightforward to observe that for our control purposes it is not necessary to check for all determinants of all possible minors in $\mathbf{J}$, but it is sufficient that at least one $n \times n$ minor of $\mathbf{J}$ has full rank in order to guarantee the system observability.

\section{PROBlem Formulation}

In order to apply the concepts of observability for the collective localization of multiple robots, we propose the leader-follower kinematic modeling together with a brief discussion about the communication network.

\section{A. Leader-follower kinematic model}

The setup we will consider throughout this work is represented in Fig. 1 and consists of $q+1$ velocity-controlled nonholonomic mobile robots whose individual kinematics can be abstracted as a unicycle model

$$
\dot{x}=v \cos \theta, \quad \dot{y}=v \sin \theta, \quad \dot{\theta}=\omega
$$

where the position is $(x, y)$ and $\theta$ represents the orientation with respect to the world frame. One of these robots is the leader $\langle L\rangle$ whose configuration vector in the world frame $\langle W F\rangle$ is $\left(x_{L}, y_{L}, \theta_{L}\right)^{T}$. The other $q$ robots are the followers $\left\langle F_{i}\right\rangle(i=1, \ldots, q)$ described by $\left(x_{F_{i}}, y_{F_{i}}, \theta_{F i}\right)^{T}$. The control inputs of both leader and followers are the translational and angular velocities $\left(v_{L}, \omega_{L}\right)^{T}$ and $\left(v_{F_{i}}, \omega_{F_{i}}\right)^{T}$, respectively.

The kinematic modeling for the setup with many follower robots will be obtained as a direct extension of the simplified case with $q=1$. The leader $\langle L\rangle$ is equipped with an omnidirectional camera and is able to observe the $i$-th follower through a colored marker $P_{i}$ placed at a distance $d$ along its translational axis (see Fig. 1) thus measuring the $i$-th follower heading direction $\xi_{i}$ with respect to the leader.

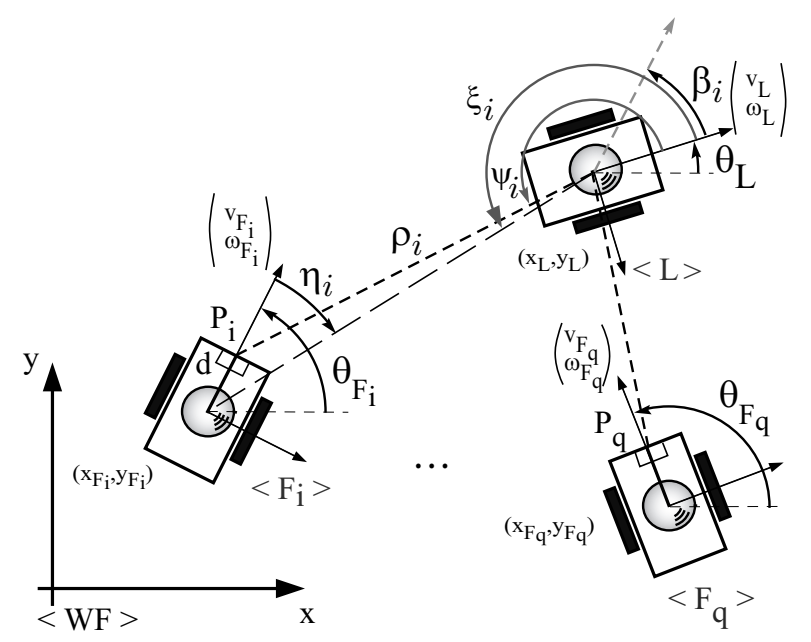

Fig. 1. Basic leader-follower setup in polar coordinate representation with panoramic cameras.
In analogous way also $\left\langle F_{i}\right\rangle$ is equipped with a panoramic camera and can measure the angle $\eta_{i}$.

In order to simplify the notation we introduce the polar coordinate representation [5]. Let $\rho_{i}$ be the length of the line of sight from the panoramic camera $\langle L\rangle$ to the marker in $P_{i}$. Let $\psi_{i}$ be the angle from the leader $y$-axis and the line of sight of $P_{i}$ and $\beta_{i}$ the relative orientation between the two robots, i.e the bearing. It is an easy matter to show that $\beta_{i}=\xi_{i}-\eta_{i}+\pi$.

Proposition 3 (One-leader-one-follower kinematics):

Under the above conditions, the one-leader-one-follower kinematics (Fig. 1) can be written in the following way:

$$
\dot{\mathbf{s}}_{i}=\mathbf{G}_{i}\left(\mathbf{s}_{i}\right) \mathbf{u}_{i}, \quad i=1, \ldots, q
$$

where

$$
\mathbf{G}_{i}=\left[\begin{array}{cccc}
\cos \gamma_{i} & d \sin \gamma_{i} & -\cos \psi_{i} & 0 \\
\frac{-\sin \gamma_{i}}{\rho_{i}} & \frac{d \cos \gamma_{i}}{\rho_{i}} & \frac{\sin \psi_{i}}{\rho_{i}} & -1 \\
0 & -1 & 0 & 1
\end{array}\right]
$$

being $\quad \mathbf{u}_{i}=\left[v_{F_{i}} \omega_{F_{i}} v_{L} \omega_{L}\right]^{T}, \quad \mathbf{s}_{i} \triangleq\left[\rho_{i} \psi_{i} \beta_{i}\right]^{T} \quad$ and $\gamma_{i} \triangleq \beta_{i}+\psi_{i}$.

Proof: Consider the setup in Fig. 1. It may be seen that $\rho_{i}=\sqrt{\rho_{i x}^{2}+\rho_{i y}^{2}}$, where $\rho_{i x} \triangleq\left(x_{L}-x_{F_{i}}-d c \theta_{F_{i}}\right)$ and $\rho_{i y} \triangleq\left(y_{L}-y_{F_{i}}-d s \theta_{F_{i}}\right)(s \theta=\sin \theta, c \theta=\cos \theta)$, whose derivative leads to:

$$
\begin{aligned}
\dot{\rho}_{i}= & \frac{1}{\rho_{i}}\left[\rho_{i_{x}}\left(\dot{x}_{L}-\dot{x}_{F_{i}}+\dot{\theta}_{F_{i}} d s \theta_{F_{i}}\right)+\right. \\
& \left.+\rho_{i_{y}}\left(\dot{y}_{L}-\dot{y}_{F_{i}}-\dot{\theta}_{F_{i}} d c \theta_{F_{i}}\right)\right]
\end{aligned}
$$

and putting the unicycle kinematics (9) in (11), after few computations we can write

$$
\begin{aligned}
& \dot{\rho}_{i}=(\underbrace{\rho_{i_{x}} c \theta_{L}+\rho_{i_{y}} s \theta_{L}}_{(a)}) \frac{v_{L}}{\rho_{i}}-(\underbrace{\rho_{i_{x}} c \theta_{F_{i}}+\rho_{i_{y}} s \theta_{F_{i}}}_{(b)}) \frac{v_{F i}}{\rho_{i}} \\
&+d(\underbrace{\rho_{i_{x}} s \theta_{F_{i}}-\rho_{i_{y}} c \theta_{F_{i}}}_{(c)}) \frac{\omega_{F_{i}}}{\rho_{i}}
\end{aligned}
$$

The factor $(a)$ is the scalar product between $\left(\rho_{i_{x}}, \rho_{i_{y}}\right)^{T}$ and $\left(c \theta_{L}, s \theta_{L}\right)^{T}$ that corresponds to $-\rho_{i} c \psi_{i}$. Analogously (b) is $-\rho_{i} c \gamma_{i}$ where $\gamma_{i}=\psi_{i}+\beta_{i}$. Note that $\beta_{i}=\theta_{L}-$ $\theta_{F_{i}}$. Moreover $(c)$ corresponds to $\rho_{i} s \gamma_{i}$. By substituting the retrieved values of $(a),(b)$ and $(c)$ into (12), we obtain the desired expression for $\dot{\rho}_{i}$

Let us now retrieve the expression for $\dot{\psi}_{i}$. From Fig. 1 it is possible to see that $\psi_{i}=\arctan \left(\rho_{i_{y}} / \rho_{i_{x}}\right)-$ $\theta_{L}$ from which the first time derivative yields to $\dot{\psi}_{i}=\frac{1}{\rho_{i_{x}}^{2}+\rho_{i_{y}}^{2}}\left[\dot{\rho}_{i_{y}} \rho_{i_{x}}-\dot{\rho}_{i_{x}} \rho_{i_{y}}\right]-\omega_{L}$ and substituting the above expressions for $\dot{\rho}_{i_{x}}$ and $\dot{\rho}_{i_{y}}$ and grouping all terms with the same velocities then it follows:

$$
\begin{aligned}
\dot{\psi}_{i} & =\frac{v_{L}}{\rho_{i}^{2}} \underbrace{\left(\rho_{i_{x}} s \theta_{L}-\rho_{i_{y}} c \theta_{L}\right)}_{\left(a^{\prime}\right)}+\frac{v_{F i}}{\rho_{i}^{2}} \underbrace{\left(\rho_{i_{y}} c \theta_{F i}-\rho_{i_{x}} s \theta_{F i}\right)}_{\left(b^{\prime}\right)}+ \\
& -\frac{\omega_{F_{i}} d}{\rho_{i}^{2}} \underbrace{\left(\rho_{i_{x}} c \theta_{F i}+\rho_{i_{y}} s \theta_{F i}\right)}_{\left(c^{\prime}\right)}-\omega_{L}
\end{aligned}
$$


and in an analogous way it is possible to show that $\left(a^{\prime}\right)$, $\left(b^{\prime}\right)$ and $\left(c^{\prime}\right)$ correspond respectively to $\rho_{i} s \psi_{i},-\rho_{i} s \gamma_{i}$ and $-\rho_{i} c \gamma_{i}$.

The results obtained in (10) are now extended to the "oneleader-many-follower" case.

Proposition 4 (One-leader-many-follower Kinematics):

Consider the one-leader-many-follower setup in Fig. 1. In this case the kinematic model can be readily retrieved as an extension of the one-leader-one-follower case (10) and is:

$$
\dot{\mathbf{s}}=\mathbf{G}(\mathbf{s}) \mathbf{u}
$$

where the state vector is $\mathbf{s}=\left[\rho_{1}, \psi_{1}, \beta_{1}, \ldots, \rho_{q}, \psi_{q}, \beta_{q}\right]^{T} \in \mathbb{R}^{3 q}, \quad \mathbf{G}(\mathbf{s}) \quad$ is as in (14), $\gamma_{i} \triangleq \beta_{i}+\psi_{i} \forall i=1, \ldots, q$ and $\mathbf{u}=\left[v_{F_{1}}, \omega_{F_{1}}, v_{F_{2}}, \omega_{F_{2}}, \ldots, v_{F_{q}}, \omega_{F_{q}}, v_{L}, \omega_{L}\right] \in \mathbb{R}^{2(q+1)}$.

$$
\mathbf{G}(\mathbf{s})=\left[\begin{array}{ccccccc}
c \gamma_{1} & d s \gamma_{1} & \ldots & 0 & 0 & -c \psi_{1} & 0 \\
-\frac{s \gamma_{1}}{\rho_{1}} & \frac{d c \gamma_{1}}{\rho_{1}} & \ldots & 0 & 0 & \frac{s \psi_{1}}{\rho_{1}} & -1 \\
0 & -1 & \ldots & 0 & 0 & 0 & 1 \\
\vdots & \vdots & \ddots & \vdots & \vdots & \vdots & \vdots \\
0 & 0 & \ldots & c \gamma_{q} & d s \gamma_{q} & -c \psi_{q} & 0 \\
0 & 0 & \ldots & -\frac{s \gamma_{q}}{\rho_{q}} & d \frac{c \gamma_{q}}{\rho_{q}} & \frac{s \psi_{q}}{\rho_{q}} & -1 \\
0 & 0 & \ldots & 0 & -1 & 0 & 1
\end{array}\right]
$$

\section{B. Distributed architecture/communication network}

We here briefly describe the communication network between the leader and the followers. In Fig. 2 we represented a scheme for the information flow. In particular we will assume that the leader transmits to the $i$-th follower the velocity control $\left(v_{F i}, \omega_{F i}\right)$ needed to bring the multi-robot configuration toward a desired value. The computation of the control law is made by the leader, together with the estimation of the state. The bearing $\beta_{i}$ is computed through the knowledge of $\eta_{i}$ that is transmitted by the $i$-th follower to the leader. To simplify the discussion we will henceforth refer

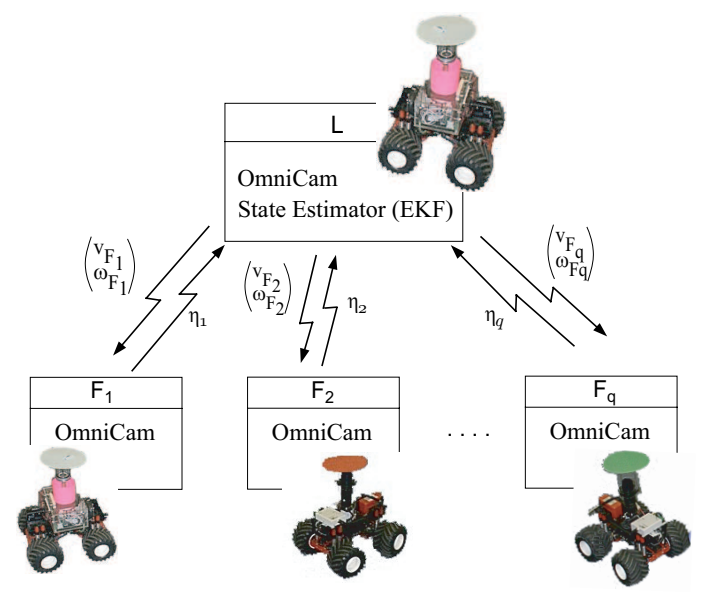

Fig. 2. Communication network architecture. All computation is carried out on the leader who receives the measurements (the angle $\eta_{i}$ ) from all the follower and transmits the control input $\left(v_{F_{i}}, \omega_{F_{i}}\right)^{T}$. only to the bearing $\beta_{i}$ implicitly assuming the transmission of $\eta_{i}$.

\section{LEADER-FOLLOWER LOCALIZABILITY}

In this section we will use the Observability Rank Condition (Prop. 2) together with the kinematic leader-follower models proposed in Sec. III-A, in order to provide an analytical description on observability in the case of mobile camera network using only bearing. We will also give a physically meaningful interpretation of it.

\section{A. Case I : one-leader-one-follower}

We here present the localizability study for the setup presented in Fig. 1 with one follower $(q=1)$. As previously discussed we suppose that the leader can observe the $i$ th follower and measure a two dimensional output vector, namely

$$
\mathbf{y}_{i} \triangleq\left[\begin{array}{ll}
y_{1} & y_{2}
\end{array}\right]^{T}=\left[\begin{array}{ll}
\psi_{i} & \beta_{i}
\end{array}\right]^{T}
$$

From Prop. 2 it may be seen that the observability of system (10) (with $i=1$ ), under the hypothesis of outputs as in (15), is guaranteed when at least one $3 \times 3$ submatrix of the whole Extended Output Jacobian $\mathbf{J} \in \mathbb{R}^{6 \times 3}$ is nonsingular. For each follower let us consider, e.g. the submatrix $\mathbf{J}_{i}$ :

$$
\mathbf{J}_{i}=\left[\begin{array}{lll}
\frac{\partial y_{1}}{\partial \rho_{i}} & \frac{\partial y_{1}}{\partial \psi_{i}} & \frac{\partial y_{1}}{\partial \beta_{i}} \\
\frac{\partial \dot{y}_{1}}{\partial \rho_{i}} & \frac{\partial \dot{y}_{1}}{\partial \psi_{i}} & \frac{\partial \dot{y}_{1}}{\partial \beta_{i}} \\
\frac{\partial y_{2}}{\partial \rho_{i}} & \frac{\partial y_{2}}{\partial \psi_{i}} & \frac{\partial y_{2}}{\partial \beta_{i}}
\end{array}\right]=\left[\begin{array}{ccc}
0 & 1 & 0 \\
\frac{\partial \dot{\psi}_{i}}{\partial \rho_{i}} & \frac{\partial \dot{\psi}_{i}}{\partial \psi_{i}} & \frac{\partial \dot{\psi}_{i}}{\partial \beta_{i}} \\
0 & 0 & 1
\end{array}\right]
$$

whose determinant is:

$$
\operatorname{det}\left(\mathbf{J}_{i}\right)=-\frac{\partial \dot{\psi}}{\partial \rho_{i}}=\frac{1}{\rho_{i}}\left[\dot{\psi}_{i}+\omega_{L}\right]
$$

It results that if $\operatorname{det}\left(\mathbf{J}_{i}\right) \neq 0$ then the state $\mathbf{s}(t)$ of the leader$i$ th follower is observable.

\section{B. Case II: one-leader-many-follower}

In the general case of $q$ followers we can adopt the whole system description in Prop. 4 to compute the Extended Output Jacobian. It is an easy matter to verify that the $3 \times 3$ structure of $\mathbf{J}_{i}$ in (16), when extended for the one leader many follower case is not singular when

$$
\frac{1}{\rho_{1} \cdots \rho_{q}}\left(\dot{\psi}_{1}+\omega_{L}\right)\left(\dot{\psi}_{2}+\omega_{L}\right) \cdots\left(\dot{\psi}_{q}+\omega_{L}\right) \neq 0 .
$$

\section{Unobservability geometrical interpretation}

We want to give here a geometrical interpretation of the singularity of (16) in the basic example depicted in Fig. 3 in which a leader observes two moving followers at different time instants $t=0$ and $t=1$. At $t=0$ the Follower \#1 has the same heading of the leader (i.e. $\beta_{1}=0$ ) while the second $(\# 2)$ has not $\left(\beta_{2} \neq 0\right)$. Then after a translational motion with equal velocity modulus, it results that $\psi_{1}$ did not change $\left(\psi_{1}(0)=\psi_{1}(1)\right.$ i.e. no temporal change in visual information), while $\psi_{2}(0) \neq \psi_{2}(1)$. From this we have that $\operatorname{det}\left(\mathbf{J}_{1}\right)=0$ while $\operatorname{det}\left(\mathbf{J}_{2}\right) \neq 0$, i.e. differently from follower $\# 2$, the state of leader $\# 1$ is not observable. 


\section{NONLINEAR OBSERVER AND FORMATION CONTROL}

In the previous section we obtained sufficient conditions for the perspective observability of a group of nonholonomic mobile robots equipped with vision sensors and moving in a leader-follower configuration. We here apply the Extended Kalman Filter (EKF) to estimate the robot configurations and we design an input-output feedback linearizing control law to make all the robots able to follow the leader.

\section{A. Observer design: Continuous-Discrete EKF}

For the sake of simplicity we assumed no model uncertainty:

$$
\dot{\mathbf{s}}(t)=\mathbf{g}(\mathbf{s}(t), t, \mathbf{u}), \quad \mathbf{s}(0)=\mathbf{s}_{0}, \quad 0 \leq t \leq \tau
$$

We moreover assume discrete measurements $\mathbf{z}\left(t_{k}\right)=\mathbf{h}\left(\mathbf{s}\left(t_{k}\right)\right)+\mathbf{v}\left(t_{k}\right)$ (with $k=1,2, \ldots, N$ ) where $\mathbf{z}\left(t_{k}\right) \in \mathbb{R}^{2 q}$ is the discrete measurement output from sensors evaluated at discrete time $t_{k} \cdot \mathbf{h}\left(\mathbf{s}\left(t_{k}\right)\right)$ is the output model and will be henceforth referred to as $\mathbf{h}_{k}$. The sensor random error $\mathbf{v}\left(t_{k}\right)$ is assumed to have gaussian distribution , i.e. $\mathbf{v}\left(t_{k}\right) \sim \mathcal{N}\left(0, \mathbf{R}\left(t_{k}\right)\right)$ where $\mathbf{R}\left(t_{k}\right)$ is the error covariance matrix. Initial condition is $\mathbf{s}(0) \sim \mathcal{N}\left(\hat{\mathbf{s}}(0), \mathbf{P}_{0}\right)$. It must be remarked that these outputs are retrieved without any need of camera model knowledge. Being $t_{k}$ the sensor sampling time and $T_{p} \triangleq T_{s} / \eta$ the microcontroller sampling time (with $\eta \in \mathbb{N}$ ), then the EKF Propagation and Update steps are

$$
\begin{aligned}
& \text { (Propagation) } \\
\mathbf{P}\left(T_{p}+1\right)= & \mathbf{P}\left(T_{p}\right)+T_{p}\left[\mathbf{F P}\left(T_{p}\right)+\mathbf{P}\left(T_{p}\right) \mathbf{F}^{T}\right] \\
\hat{\mathbf{s}}\left(T_{p}+1\right)= & \hat{\mathbf{s}}\left(T_{p}\right)+T_{p} \mathbf{g}\left(\hat{\mathbf{s}}\left(T_{p}\right), T_{p}, \mathbf{u}\right) \\
& (\text { Update }) \\
\mathbf{K}\left(t_{k}\right)= & \mathbf{P}^{-}\left(t_{k}\right) \mathbf{H}^{T}\left[\mathbf{H P}^{-}\left(t_{k}\right) \mathbf{H}^{T}+\mathbf{R}\right]^{-1} \\
\mathbf{P}\left(t_{k}\right)= & \left(\mathbf{I}-\mathbf{K}\left(t_{k}\right) \mathbf{H}\right) \mathbf{P}^{-}\left(t_{k}\right) \\
\hat{\mathbf{s}}\left(t_{k}\right)= & \hat{\mathbf{s}}^{-}\left(t_{k}\right)+\mathbf{K}\left(\mathbf{z}_{t_{k}}-\mathbf{h}_{k}\left(\hat{\mathbf{x}}^{-}\left(t_{k}\right)\right)\right)
\end{aligned}
$$

\section{B. Controller design}

In the spirit of [5] the control strategy to move the sensor formation from an initial towards a target configuration w.r.t. the leader has been designed via an inputstate feedback linearizing control law. Note that in the leader-to-follower control $\beta_{i}$ is controlled to 0 as $\rho_{i}$ and

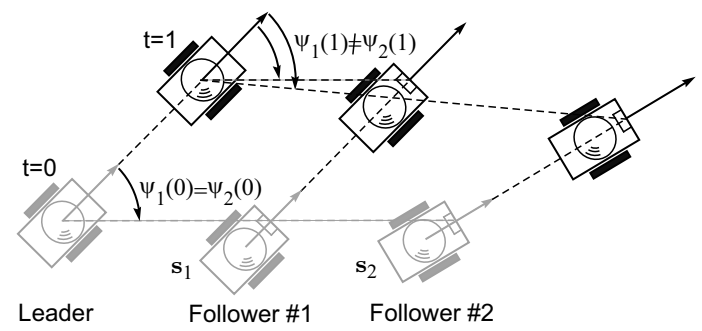

Fig. 3. Geometrical interpretation for the indistinguishability and singularity of observability matrix. $\psi_{i}$ approach to the desired values. This lead us to consider a reduced state vector $\mathbf{s}_{r}=\left[\rho_{1}, \psi_{1}, \ldots \rho_{q}, \psi_{q}\right]^{T}$ and $\mathbf{s}_{r}^{d e s}=\left[\rho_{1}^{d e s}, \psi_{1}^{d e s}, \ldots, \rho_{q}^{\text {des }}, \psi_{q}^{\text {des }}\right]^{T}$. Moreover the kinematic model as in (10) can also be written as $\dot{\mathbf{s}}_{r}=\mathbf{H}\left(\mathbf{s}_{r}\right) \mathbf{u}_{F}+$ $\mathbf{F}\left(\mathbf{s}_{r}\right) \mathbf{u}_{L}$ where $\mathbf{u}_{F} \triangleq\left[v_{F_{1}}, \omega_{F_{1}}, \ldots, v_{F_{q}}, \omega_{F_{q}}\right]^{T}$ and $\mathbf{u}_{L}=$ $\left[v_{L}, \omega_{L}\right]^{T}$, and $\mathbf{H}\left(\mathbf{s}_{r}\right)$ is made of the first $2 q$ columns of $\mathbf{G}\left(\mathbf{s}_{r}\right)$. Moreover $\mathbf{F}\left(\mathbf{s}_{r}\right)$ is made of the last two columns of $\mathbf{G}\left(\mathbf{s}_{r}\right)$. Note that due to the above considerations on $\beta_{i}$ the rows corresponding to $\dot{\beta}_{i}$ are not considered in $\mathbf{H}\left(\mathbf{s}_{r}\right)$ and $\mathbf{F}\left(\mathbf{s}_{r}\right)$.

The linearizing control input $\mathbf{u}_{F}$ to the followers is

$$
\mathbf{u}_{F}=\mathbf{H}^{-1}\left(\mathbf{s}_{r}\right)\left(\mathbf{p}-\mathbf{F} \mathbf{u}_{L}\right)
$$

where $\mathbf{p}$ is an auxiliary control input given by $\mathbf{p}=\mathbf{K}\left(\mathbf{s}_{r}^{\text {des }}-\mathbf{s}_{r}\right)$ where the control gain matrix $\mathbf{K}$ is:

$$
\mathbf{K}=\left[\begin{array}{ccccc}
k_{1}^{1} & 0 & \ldots & 0 & 0 \\
0 & k_{1}^{2} & \ldots & 0 & 0 \\
\vdots & \vdots & \vdots & \vdots & \vdots \\
0 & 0 & \ldots & k_{q}^{1} & 0 \\
0 & 0 & \ldots & 0 & k_{q}^{2}
\end{array}\right]
$$

Note that to guarantee the asymptotic convergence we must ensure that $k_{i}^{1}, k_{i}^{2}>0(\forall i=1, \ldots, q)$.

\section{Simulations}

In this section, we present results of the estimation of position and orientation of one or more followers with respect to a leader, using an Extended Kalman Filter. We simulate the leader-follower control law (19). We present some results for the case in which the input-output linearizing control law proposed in Sec.V-B is applied, in order to make all robots (in this case two $q=2$ ) able to follow the leader. In the first case the leader is moving on a straight line and the desired relative pose of a follower with respect to leader is $\rho_{1,2}^{d e s}=$ $0.5 \mathrm{~m}, \psi_{1}^{\text {des }}=3 / 4 \pi \mathrm{rad}$ and $\psi_{2}^{\text {des }}=5 / 4 \pi \mathrm{rad}$. We assumed an initial uncertainty of $1 m$ on $\rho_{1}(0)$ and measurement noise of $\sigma=1 \mathrm{deg}$. Robot trajectories are showed in Fig. 4(a). Both estimation and trajectory errors converge to zero (Fig. 4(b)(f)). In the second case the leader is moving with velocities $\left(v_{L}, \omega_{L}\right)=(2, \cos (t) / 2)$. The same uncertainties than above are assumed. Robot trajectories are showed in Fig. 5(a). Also in this case all estimation and trajectory errors converge to zero (Fig. 5(b)-(f)).

\section{CONCLUSIONS AND FUTURE WORK}

We have presented the localization problem for a camera network and we solved it in the case of cameras mounted on mobile nonholonomic robots moving in leader-follower formations, using only bearing. In particular we proved a sufficient condition for observability and we showed that recursive estimation enables a leader-follower formation if the leader is not stuck in an unobservable set-up. Please remember that the leader-follower control here presented is only based on the visual information provided by the observation of colored blobs on the robots. Future research lines include (1) better understanding of closed-loop control 


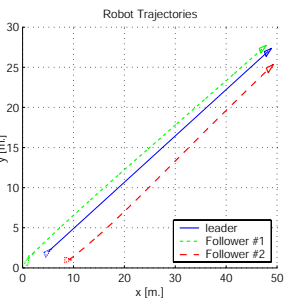

(a)

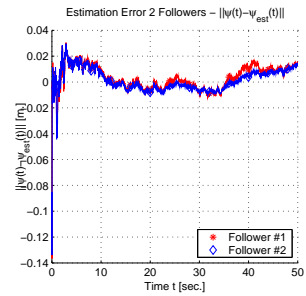

(c)

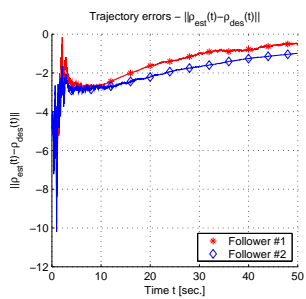

(e)

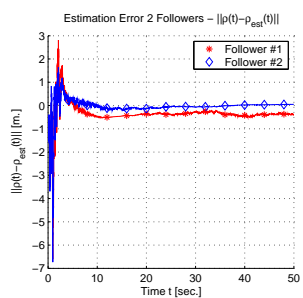

(b)

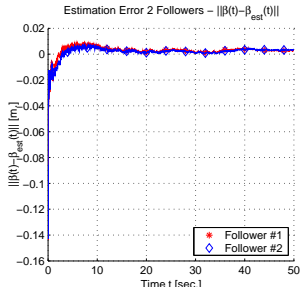

(d)

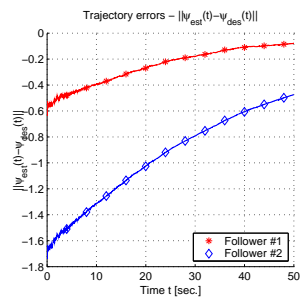

(f)

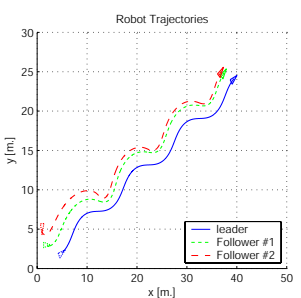

(a)

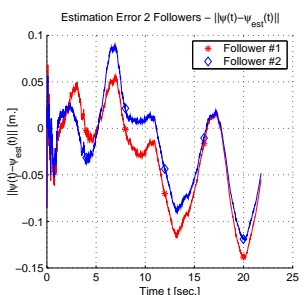

(c)

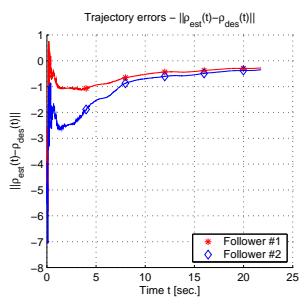

(e)

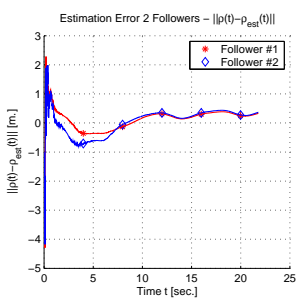

(b)

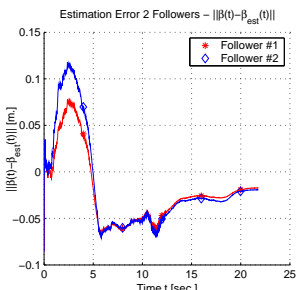

(d)

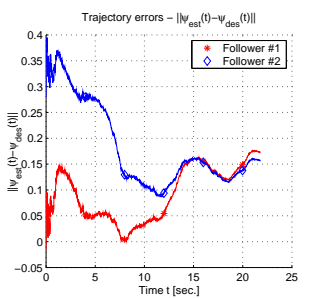

(f)
Fig. 4. (Leader moving on a straight line) Estimation and trajectory errors in the case of leader-to-followers stabilization control.

effects on the observer performances, (2) the design of a control law that selects inputs which minimize the uncertainty of the location estimates, (3) the investigation of collective localization with minimal communication between agents, (4) use of estimators like Unscented Kalman filters which are less dependent on the assumption of Gaussian distributions.

\section{REFERENCES}

[1] S. Aranda, S. Martinez, and F. Bullo. On optimal sensor placement and motion coordination for target tracking. In IEEE Conference in Robotics and Automation, Barcelona, Spain, April 2005. (in press).

[2] A. Bicchi, D. Prattichizzo, A. Marigo, and A. Balestrino. On the observability of mobile vehicles localization. In Proc. IEEE Mediterrean Conf. on Control and Systems, 1998.

[3] K.K. Chintalapudi, A. Dhariwal, R. Govindan, and G.S. Sukhatme. Ad-hoc localization using ranging and sectoring. In IEEE INFOCOM 2004, Hong Kong, 2004.

[4] F. Conticelli, A. Bicchi, and A. Balestrino. Observability and nonlinear observers for mobile robot localization. In IFAC Int. Symp. on Robot Control, SyRoCo 2000.

[5] A.K. Das, R. Fierro, V. Kumar, J.P. Ostrowsky, J. Spletzer, and C. Taylor. A vision-based formation control framework. IEEE Robotics and Automation, Trasaction on, 18(5):813-825, October 2002.

[6] L. Doherty, L.E. Ghaoui, and S.J. Pister. Convex position estimation in wireless sensor networks. In IEEE Infocom, volume 3, pages 16551663, April 2001.

[7] R. Grabowski and P. Khosla. Localization techniques for a team of small robots. In IEEE/RSJ International Conference on Intelligent Robots and Systems, Maui, Hawaii, 2001.
[8] R. Hermann and A. Krener. Nonlinear controllability and observability. IEEE Transactions on Automatic Control, AC-22:728-740, 1977.

[9] J.P. Hespanha. State estimation and control for systems with perspective outputs. In 41st IEEE Conference on Decision and Control, volume 2, pages $2208-2213,2002$.

[10] H. Inaba, A. Yoshida, R. Abdursul, and B.K. Ghosh. Observability of perspective dynamical systems. In IEEE Conference on Decision and Control, pages 5157-5162, 2000.

[11] H. Inaba, A. Yoshida, R. Abdursul, and B.K. Ghosh. Observability of Perspective Dynamical Systems. In IEEE 39th Conf. on Decision and Control, Sydney, Australia, pages 5157-5162, December 2000.

[12] A. Isidori. Nonlinear Control Systems. Springer, 3rd edition edition, London, UK 1995

[13] A.J. Krener and W. Respondek. Nonlinear observers with linearizable error dynamics. SIAM Journal on Control and Optimization, 23:197216, 1985.

[14] D. Moore, J. Leonard, D. Rus, and S. Teller. Robust distributed network localization with noisy range measurements. In Proceedings of the Second ACM Conference on Embedded Networked Sensor Systems (SenSys '04), Baltimore, MD, 2004.

[15] D. Niculescu and B. Nath. Ad hoc positioning system (APS) using AOA. In INFOCOM 2003. 22nd Annual Joint Conference of the IEEE Computer and Communications Societies, volume 3, pages 1734 1743, April 2003.

[16] H. Nijmeijer and A.J. van der Shaft. Nonlinear dynamical control systems. Springer, 1990.

[17] S. Teller, M. Brand, and M. Antone. Spectral solution of largescale extrinsic camera calibration as a graph embedding problem,. In European Conference on Computer Vision (ECCV), 2003 